\title{
Variance Homogeneity Test Based on Cumulative Wavelet Coefficients
}

\author{
Abdeslam Serroukh ${ }^{1, *}$, Khudhayr A. Rashedii ${ }^{2,3}$ \\ ${ }^{1}$ Polydisciplinary Faculty of Larache, University Abdelmalek Essaadi, Morocco \\ ${ }^{2}$ College of Sciences, University of Hail, Saudi Arabia \\ ${ }^{3}$ School of Mathematical Sciences, University Sains Malaysia, 11800, Minden, Malaysia
}

Copyright $@ 2020$ by authors, all rights reserved. Authors agree that this article remains permanently open access under the terms of the Creative Commons Attribution License 4.0 International License

\begin{abstract}
The aim of this paper is to address the problem of variance break detection in time series in wavelet domain. The maximal overlapped discrete wavelet transform (MODWT) decomposes the series variance across scales into components known as the wavelet variances. We introduce all scale wavelet coefficients based test statistic that allows detecting a break in the homogeneity of the variance of a series through changes in the mean of wavelet variances. The statistic makes use of the traditional CUSUM (cumulative sum) based test designed to test for a break in the mean and constructed using cumulative sums of the square of wavelet coefficients. Under moments and mixing conditions, the test statistic satisfies the functional central limit theorem (FCLT) for a broad class of time series models. The overall performance of our test statistic is compared to the traditional Inclan [8] test statistic. The effectiveness of our statistic is supported by good performances reported in simulations and is as reliable as the traditional statistic. Our method provides a nonparametric test procedure that can be applied to a large class of linear and non linear models. We illustrate the practical use of our test procedure with the quarterly percentage changes in the Americans personal savings data set over the period 1970-2016. Both statistics detect a break in the variance in the second quarter of 2001.
\end{abstract}

Keywords Cumulative Wavelet Variance, Maximal Overlaped Discrete Wavelet Transform, Test for Homogeneity of Variance, Time Series

\section{Introduction}

The test problem that addresses the issue of change in the variance homogeneity of time series has received considerable attention in the literature. Most of the available procedure have developed tests that make use of cumulative sums of squares (CUSUMs) principale to test for breaks in the variance. From a methodological point of view, the test statistics developed for variance change detection in the case of independent and identically distributed (i.i.d) random variables setting as in [7] and [8] may not work in the time series setup and suitable modification were needed to account for the temporal dependence in the data. Many different approaches for addressing such test problem have been developed; see, for example [4], [7], [15], and [13]. The properties of the classical CUSUMs statistic in the variance break detection in general setting were examined by several authors. We can cite for example [6] and [9] for ARCH models, [11] for standardized residuals from an estimated GARCH model, [15] for a class of nonstationary and nonparametric time series models, [4] for ongoing monotring process and [10] for detecting changes in general autocovariance structure in nonstationary time series using locally stationary wavelet process. Our approach is inspired by the application of the discrete wavelet transform in [3] for testing homogeneity of variance in a time series with long memory structure. Here we limit ourselves to test statistics based on the cumulative sums of squares of wavelet coefficients and take a different approach to investigate the null hypotheses of no break in the variance. We are motivated by the fact that the sample variance of a time series can be decomposed into components known as the wavelet variances each of which is associated with a particular scales as in [17]. This decomposition can be achieved through the use of the maximal-overlap discrete wavelet transform (MODWT) also called the undecimated or shift invariant discret wavelet transform, and has been discussed in the wavelet literature, see [1-3], and [12]. In a similar manner the variance $\sigma_{X}^{2}$ of $X_{t}$ is decomposed into variances of wavelets and scaling coefficients as given by equation (6) in [16]. In theory any changes in either the variance of wavelets or scaling coefficients would directly results in the shift of $\sigma_{X}^{2}$. On the other hand and in constract to the scaling coefficients the wavelet coefficients 
demean and remove trends from a time series. These attractive characteristics give us a motivation in this work to explore the cumulative sums of the squared wavelet coefficients which is a time dependent series designed to track the build up over time of the sample variance across scales up to a fixed level.

This paper is orgonized as follows. Section 1 provides an introduction to our test problem and a brief review of the MODWT tranform in the literature. Section 2 introduces the definition of the statistics designed to test for a break detection in the variance. In section 3 we derive the asymptotic distribution of the test statistic. Monte Carlo experiments are presented and discussed in section 4 . In section 5 we apply the test procedure to the real data series US personnal savings. Section 6 provides some conclusions.

Let $\mathbf{X}=\left(X_{0}, \cdots, X_{N-1}\right)$ be an observed time series for which we want to test for homogeneity of variance. Assume that $\mathbf{X}$ is regarded under $H_{0}$ as a realization of a second order stationary discrete time stochastic process with constant variance.

The null hypothesis of our test problem would be

$$
H_{0}: \operatorname{Var}\left(X_{t}\right)=\sigma_{X}^{2} \quad t=0, \cdots, N-1
$$

which we whish to test against the alternative hypothesis

$$
H_{1}: \operatorname{Var}\left(X_{t}\right)=\sigma_{1}^{2} \neq \sigma_{2}^{2} \quad \text { for } \quad t \geq k_{0} \text {, where } k_{0}<<N
$$

the times of variance change $k_{0}$ is unknown.

\subsection{The MODWT transform}

The maximal-overlap discrete wavelet transform (MODWT) is variant of the standard discrete wavelet transform (DWT) and has been discussed in the wavelet literature see [1-3]. For the class of discrete compactly supported Daubechies wavelets, we denote $\left\{\tilde{h}_{j, l}\right.$ and $\left.\tilde{g}_{j, l}, l=0, \cdots, L_{j}-1\right\}$ the level $\mathrm{j}$ of a wavelet and scaling filters of length $L_{j}=\left(2^{j}-1\right)(L-1)+1$. These filters are discussed and given in more details in [3]. The stochastic processes resulting from applying these filters to $\left\{X_{t}\right\}$ are respectively given by the level $j$ wavelet and scaling coefficients

$$
W_{j, t}=\sum_{l=0}^{L_{j}-1} \tilde{h}_{j, l} X_{t-l}, \quad V_{j, t}=\sum_{l=0}^{L_{j}-1} \tilde{g}_{j, l} X_{t-l} \quad j=1, \cdots J
$$

The MODWT wavelet and scaling coefficient based on a finite sample size and run up to a maximum level $\mathbf{J}$ are given for $t=0,1, \cdots N-1$ by

$$
\widetilde{W}_{j, t}=\sum_{l=0}^{L_{j}-1} \tilde{h}_{j, l} X_{t-l \bmod N} \quad \widetilde{V}_{j, t}=\sum_{l=0}^{L_{j}-1} \tilde{g}_{j, l} X_{t-l \bmod N}
$$

obtained as a result of circularly filtering $X_{0}, X_{1}, \cdots, X_{N-1}$ with the filters $\left\{\tilde{h}_{j, l}\right\}$ and $\left\{\tilde{g}_{j, l}\right\}$ where $X_{t \bmod N}=X_{t}$ if $t \geq$ 0 and $X_{t \bmod N}=Y_{N-|t|}$ if $t<0$. Note that in order to obtain the time in wavelet coefficients aligned with time is $X_{t}$ we circularly shift these coefficients by an amount dictated by the phase properties of the filter been used as in [3] page 198.

\section{Cumulative wavelet variance}

The wavelet transform decomposes the variance $\sigma_{X}^{2}$ into finite number of cumulative wavelet variances across scales and a scaling variance.

$$
\sigma_{X}^{2}=\sum_{j=1}^{\infty} \sigma_{W_{j}}^{2}=\sum_{j=1}^{J} \sigma_{W_{j}}^{2}+\sigma_{V_{J}}^{2}
$$

where $\sigma_{W_{j}}^{2}=\operatorname{Var}\left(W_{j, t}\right)$ and $\sigma_{V_{j}}^{2}=\operatorname{Var}\left(V_{j, t}\right)$ Any break in the variance of $X_{t}$ would result in a break of the variances of wavelet and scaling coefficients, for more details see page 190 of [17]. Therefore a break in the variance of either $W_{j, t}$ or $V_{j, t}$ would be regarded as directly related to a break in the variance of the original series. Because of the attractive properties of the wavelet coefficients, a statistical test based on these coefficients would provide an alternative method to variance break detection in time series.

Given a time series $X_{t}, t=0, \cdots, N-1$ and the transform $\widetilde{W}_{j, t}$, we then form the cumulative wavelet variance time series

$$
\widetilde{C}_{J, t}=\sum_{j=1}^{J} \widetilde{W}_{j, t}^{2} \quad t=0,1, \cdots N-1
$$

Note that $E\left(\widetilde{W}_{j, t}\right)=0$, so that

$$
E\left(\widetilde{C}_{J, t}\right)=\sum_{j=1}^{J} E\left(\widetilde{W}_{j, t}^{2}\right)=\sum_{j=1}^{J} \operatorname{Var}\left(\widetilde{W}_{j, t}\right)
$$

The mean of $\widetilde{C}_{J, t}$ is regarded as the overall contribution to the true variance of $X_{t}$ by wavelet coefficients under the null hypothesis. We will refer to the quantity $\widetilde{C}_{J, t}$ as the cumulative sums of the squared wavelet coefficients or simply the cumulative wavelet variance. From (7) it follows that any changes occurring in the variance of wavelet coefficients $\widetilde{W}_{j, t}$ is equivalent to a shift in the mean of $\widetilde{C}_{J, t}$. Therefore our test problem for homogeneity of variance of $X_{t}$ will be regarded as a test problem of detecting change in the mean of $\widetilde{C}_{J, t}$. On the other hand the contribution to the variance of $X_{t}$ by the scaling coefficients is given by $\operatorname{Var}\left(\widetilde{V}_{J, t}\right)$, and any break of this variance would reveal as well a break in $\sigma_{X}^{2}$. Both series $\widetilde{C}_{J, t}$ and $\widetilde{V}_{J, t}^{2}, t=0,1, \cdots$ are positive, but the series $\widetilde{C}_{J, t}$ is a nondecreasing sequence and can easily track the manner in which the sample variance of $X_{t}$ is building. The mean focus of this work will be to construct a wavelet coefficients based statistic to test the hypothesis of homogeneity of variance of $X_{t}$.

\subsection{Variance change point statistics}

In order to test for a change in the mean a class of commonly used test statistics is based on the CUSUM process. In our setting the wavelet based test statistic is given by

$$
T_{C, J}(k)=\frac{1}{\sqrt{N}} \sum_{t=0}^{k}\left(\widetilde{C}_{J, t}-\overline{C_{J}}\right) \quad k=1, \cdots, N-1 .
$$

where $\overline{C_{J}}=\frac{1}{N} \sum_{t=0}^{N-1} \widetilde{C}_{J, t}$. These scale related statistics are evaluated up to the physical scale $\tau_{J}=2^{J} \delta$ where $\delta$ is 

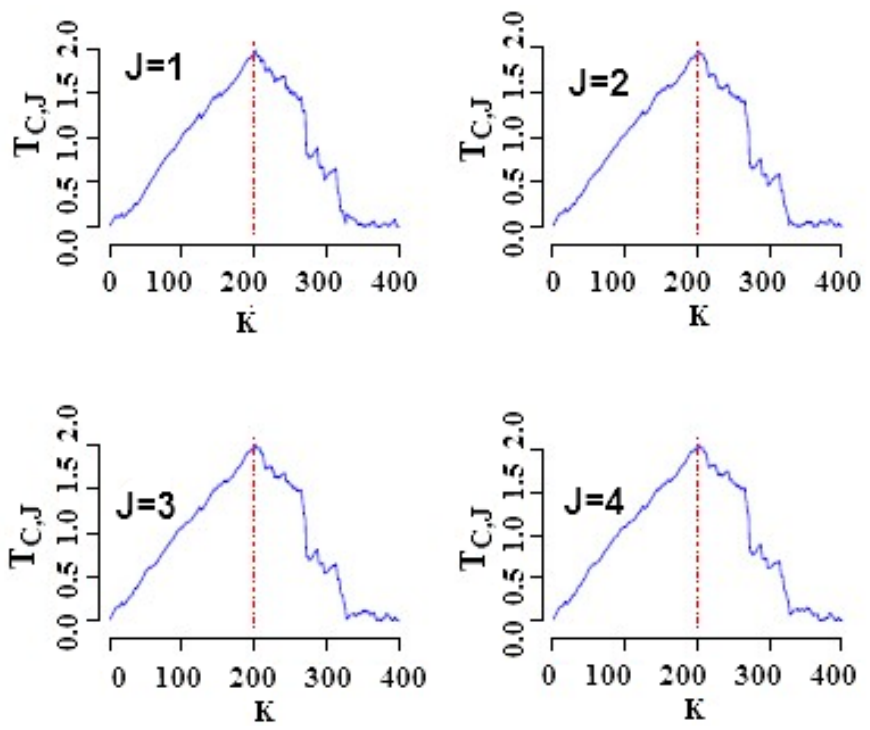

Figure 1. Plot of the statistic $T_{C, J}(k) / \hat{\sigma}_{Z}$ for $J=1,2,3,4$ for the Gaussian model $\epsilon_{t} \sim \operatorname{iidN}\left(0, \sigma_{\epsilon}^{2}\right)$ under $H_{1}$ for a single replication with $N=400$ and $k_{0}=200$. The vertical dashed line is the break location.

the sampling time between observations which equals 1 in our case. Note that this statistics contains affected coefficients in the modwt given by $\widetilde{W}_{j, t}^{2}$ for $t<L_{j}-1$. We can easily show that in term of probability their effect on our statistics is negligible

The standard test statistic for change in the variance proposed in $[8]$ is equivalent to

$$
T_{N}=\frac{1}{\sqrt{N}} \max _{1 \leq k \leq N}\left|\sum_{t=1}^{k} X_{t}^{2}-\frac{k}{N} \sum_{t=1}^{N} X_{t}^{2}\right|
$$

Under the assumption that $X_{t}, t=1, \cdots, N$ is a sequence of independent and identically distributed (i.i.d) normal random variables. This assumption was later relaxed to allow for various form of dependence and heterogeneity in $X_{t}$, see [7], [10] and [13]. The limiting distribution of this statistic depend on the long-run variance $\sigma^{2}=\sum_{k=-\infty}^{\infty} \gamma_{k}$ where $\gamma_{k}$ is the k-th order autocovariance of the series $X_{t}^{2}$ which should be estimated. Under general dependence we suggest to use the Heteroskedasticity and Autocorrelation Consistent (HAC) estimator and use the popular Bartlett kernel. $\hat{\sigma}^{2}=$ $\hat{\gamma}_{0}+2 \sum_{k=1}^{q} w_{k}(q) \hat{\gamma}_{k}$ where $w_{k}(q)=1-k /(q+1)$ are the Bartlett weights and $\hat{\gamma}_{k}$ are the sample autocovariances of the series $X_{t}^{2}$. In the case of i.i.d normal random variables $N\left(0, \sigma^{2}\right)$, all the autocovariances are such that $\hat{\gamma}_{k}=0$ for $k \neq 0$ and the estimator reduces to $\hat{\sigma}^{2}=2 \sigma^{4}$.

In the next section we establish the functional central limit theorem (FCLT) and derive the asymptotic distribution of the statistic $T_{C, J}$ for a broad class of processes under moments and mixing conditions.

In the following, $\left\{B_{0}(r) ; 0 \leq r \leq 1\right\}$ is the standard Wiener process (Brownian motion) and $\{B(r) ; 0 \leq r \leq 1\}$ is the Brownian bridge $B(r)=B_{0}(r)-r B_{0}(1)$. By $\Rightarrow$ we denote the weak convergence of random variables

\section{Asymptotic properties}

Let $\mathcal{M}_{m}^{n}(X)$ denote the $\sigma$-algebra generated by $\left\{X_{m}, \cdots, X_{n}\right\}$. The process $\left\{X_{t}\right\}$ is said to be strong mixing according to Rosenblat[14], if the mixing coefficient $\left.\alpha_{X}(n)=\sup _{A \in \mathcal{M}_{-\infty}^{0}, B \in \mathcal{M}_{n}^{\infty}} \mid P(A B)-P(A) P(B)\right) \mid \rightarrow$ 0 as $n \rightarrow \infty$

\subsection{Assumptions}

(A1) $E\left(X_{t}\right)=0$ and $E\left(X_{t}^{2}\right)=\sigma_{X}^{2}<\infty$

(A2) $\sup _{t}\left|X_{t}\right|^{\beta}<\infty$ for $\beta>4$,

(A3) $\left\{X_{t}\right\}$ is $\alpha$-mixing such that $\sum_{n=1}^{\infty}\left(\alpha_{X}(n)\right)^{1-2 / \beta}<\infty$

Lemma 1

Let $Z_{J, t}=C_{J, t}-E\left(C_{J, t}\right)$ where $C_{J, t}=\sum_{j=1}^{J} W_{j, t}^{2}$. Then under the null $H_{0}$ and assumptions (A1), (A2) and (A3)

$$
\begin{gathered}
E\left(Z_{J, t}\right)=0 \text { and } E\left(Z_{J, t}^{2}\right)<\infty \\
\sup _{t} E\left|Z_{J, t}\right|^{\beta}<\infty \\
\alpha_{Z_{J}}(k) \leq J \max _{j \leq J} \alpha_{W_{j}}(k) \\
E\left(\frac{1}{N}\left(\sum_{t=0}^{N-1} Z_{J, t}\right)^{2}\right) \rightarrow \sigma_{Z_{J}}^{2}<\infty \text { as } N \rightarrow \infty .
\end{gathered}
$$

where $\alpha_{Z_{J}}(k)$ and $\alpha_{W_{j}}(k)$ are respectively the mixing coeffcients for $Z_{J, t}$ and $W_{j, t}$.

Proof: see Appendix.

Results (9)-(12) of lemma 1 show that conditions of Corolloray 1 of Herrndof [5] are satisfied from which follow the following FCLT.

\section{Proposition 1}

Assume that conditions (A1)-(A3) are satisfied. Then under the null $H_{0}$ the statistics

$$
T_{C, J}=\max _{L_{J}-1 \leq k \leq N} \frac{1}{\hat{\sigma}_{Z_{J}}}\left|T_{C, J}(k)\right|
$$

is such that $T_{C, J} \Rightarrow \sup _{r \in[0.1]}|B(r)|$ as $N \rightarrow \infty$

The limiting distribution of the statistic $T_{C, J}$ depend on the long-run variances $\sigma_{Z_{J}}^{2}$ which is unknown and is estimated by

$$
\hat{\sigma}_{Z_{J}}^{2}=\hat{\gamma}_{Z, 0}+2 \sum_{k=1}^{q} w_{k}(q) \hat{\gamma}_{Z, k}
$$

where $\hat{\gamma}_{Z, k}$ are the sample autocovariances of the series $Z_{J, t}$. Note that as an alternative to $\hat{\gamma}_{Z, k}$ we can use the nonparametric estimator $2 \pi f_{Z_{J}}(0)$, where $f_{Z_{J}}(0)$ is the multitaper estimator of the spectrum of $Z_{J, t}$ at zero frequency. On the other hand it has been shown in [11] and [15], that under fairly mild regularity conditions that the statistic $T_{N} / \hat{\sigma}$ converges weakly in distribution to $\sup |B(r)|$ as $N \rightarrow \infty$.

$$
r \in[0.1]
$$


As suggested early the test problem of break detection in $X_{t}$ can be formulated in term of the statistic $T_{C, J}$ which aims to test for a break in the mean of the cumulative wavelet variance $\widetilde{C}_{J, t}$. Therefore any mean break detection computed by the statistic $T_{C, J}$ will be regarded as a break in the variance of $X_{t}$. In order to identify potential occurrence of change in variance for a given sample of time series $X_{0}, \cdots, X_{n-1}$, the statistic $T_{C, J}$ is computed at accumulated scales of the MODWT transform. Both statistics are computed and compared to the same critical value. For instance, for a level of significance $\alpha=5 \%$ for our test, the $95 \%$ quantile value is $q_{\alpha}=1.358$. A large value of any statistic would strongly indicates the existence of variance change. .

\section{Simulations}

We considere four different simulated models, a Gaussian $X_{t}=\epsilon_{t}$ model, a Moving average $M A(1)$ model, an autoregressive $A R(1)$ model, and the nonlinear model $X_{t}=\epsilon_{t}^{2}-1$ as given in the example below. For each model the variance is set to change at time $k_{0}$ from $\sigma_{1}^{2}$ to $\sigma_{2}^{2}$. The MODWT transform was run up to level $J=4$ with the Least Asymetric wavelet filter $L A(8)$ of length $L=8$. The choice of the wavelet filter was arbitrary with moderate length. For each model and for each sample size, the statistics $T_{N}$ and $T_{C, J}$ are then calculated and compared to the critical value $q_{\alpha}$. This process of generating data sets, running the above procedure separately for each model and then testing at significance level $\alpha=5 \%$ was repeated a total of $n=1000$. The rejection rate of the statistic $T_{C, J}$ are tabulated from level $J=1$ of the MODWT up to level $J=4$. At each level $J$ we have a test statistic $T_{C, J}$, and if we assume that at that level we reject $H_{0}$, then we have a strong evidence to claim that there is a break in the variance of $X_{t}$ at unknown change point. For example table 1 provide the simulation setting correspending to all four models.

\subsection{Example}

We consider three linear models $\epsilon_{t}$ (Gaussian) and $M A(1)$, an $A R(1)$, and a nonlinear model $X_{t}$ each with variance break occuring respectively at times $k_{0}=200$ for $N=400$ and $k_{0}=400$ for $N=800$

$\sigma_{X}^{2}=\sigma_{1}^{2}$ for $0 \leq t<k_{0} ; \quad \sigma_{X}^{2}=\sigma_{2}^{2}$ for $k_{0} \leq t \leq N-1$

In order to assess the performance of our statistic we computed the rejection rate of the statistic $T_{C, J}$ for accumulated level of scales $J=1,2,3,4$ separetly for all models and for two different sample sizes. The results are summurized in tables 2 , and show that the overall performance in term of rejection rate of the statistic $T_{C, J}$ for all models are as good as the statistic $T_{N}$ except for the AR(1) model at level $j=3$. A large value of the scale related statistic $T_{C, J}$ at some given level $J$ should be interpreted as evidence against the $H_{0}$. For example for the AR(1) model, at level $j=3$ the rejection rate was $11.8 \%$ and $12.0 \%$ which are very low for both sample sizes. But at levels $J=1$ the rejection rate is very high. Therefore caution should be taken when a low values of the statistic show up at some scales.

The important result we derive from these simulations is that our test problem for shift in the variance of $X_{t}$ is tranformed into a wavelet based test for shift in the mean of cumulative wavelet variances.
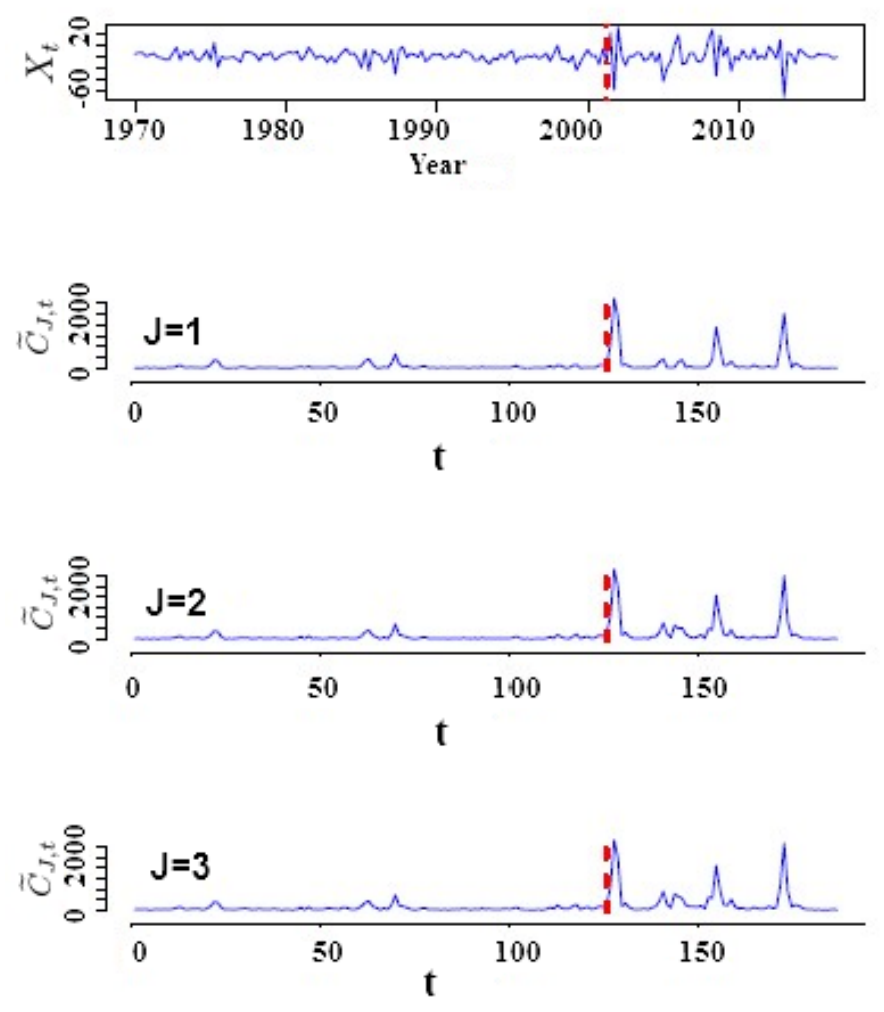

Figure 2. Panels from top to bottom are the plot of US Savings series $X_{t}$ and its wavelet cumulative variance $\widetilde{C}_{J, t}$ for $J=1,2,3$.

\section{US personnal savings}

Let us now illustrate the performance of our test statistic using real data plotted in figure 2 top panel. The data series represents the percentage changes in quarterly of the Americans personal savings which is the the percentage of their disposable income that people save after spending money and paying taxes over the period $1970-2016$. The series is of length $N=187$ and is availabe as part of the R package fpp 2 and can be loaded using uschange[,"Savings"]. The graph of the series shows that there is a high variability over the periods $2001-2010$ and $2012-2014$. The main question is to test whether there is any evidence for a break in the variance starting in the year 2001. The computed values of the test statistics $T_{C, J}$ and $T_{N}$ are shown in table 3 . At the $1-\alpha=95 \%$ level of significance the wavelet based test statistics reject the null hypothesis for levels $J=2,3,4$. The result is also comfirmed by the test statistic $T_{N}$ computed without doing the wavelet transform. This provide a strong evidence for a change in the variance in 2001 . Figure 3 shows that the series $T_{C, 1}(k)$ asso- 
Table 1. Simulated models

\begin{tabular}{|ccc|}
\hline Model & \multicolumn{2}{c|}{ variance } \\
& $t<k_{0}$ & $t \geq k_{0}$ \\
\hline (a) Gaussian: $X_{t}=\epsilon_{t}$ & $\sigma_{1}^{2}=1.0$ & $\sigma_{2}^{2}=3.0$ \\
(b) MA(1): $\eta_{t}=a \eta_{t-1}+\epsilon_{t}$ & & \\
with $\sigma_{\eta}^{2}=\left(1+a^{2}\right) \sigma_{\epsilon}^{2}$ & $\sigma_{1}^{2}=1.25$ & $\sigma_{2}^{2}=3.28$ \\
(c) AR(1): $X_{t}=\phi X_{t-1}+\epsilon_{t}$ & & \\
with $\sigma_{X}^{2}=\sigma_{\epsilon}^{2} /\left(1-\phi^{2}\right)$ & $\sigma_{1}^{2}=1.19$ & $\sigma_{2}^{2}=3.60$ \\
(d) Nonlinear: $X_{t}=\epsilon_{t}^{2}-1$ & $\sigma_{1}^{2}=1.0$ & $\sigma_{2}^{2}=3.0$ \\
\hline
\end{tabular}

Table 2. Rejection rate in percentage for model (a)-(d)

\begin{tabular}{|c|c|c|c|c|c|}
\hline & $j=1$ & $j=2$ & $j=3$ & $j=4$ & $X_{t}$ \\
\hline Model (a) & & & & & \\
$N=400$ & 99.6 & 100.0 & 100.0 & 100.0 & 100.0 \\
$N=800$ & 100.0 & 100.0 & 100.0 & 100.0 & 100.0 \\
Model (b) & & & & & \\
$N=400$ & 99.5 & 100.0 & 100.0 & 100.0 & 100.0 \\
$N=800$ & 100.0 & 100.0 & 100.0 & 100.0 & 100.0 \\
Model (c) & & & & & \\
$N=400$ & 84.0 & 55.7 & 11.8 & 26.5 & 90.8 \\
$N=800$ & 100.0 & 85.5 & 12.0 & 69.5 & 99.8 \\
Model (d) & & & & & \\
$N=400$ & 90.6 & 98.2 & 98.8 & $97 . .0$ & 94.5 \\
$N=800$ & 99.6 & 100.0 & 100.0 & 100.0 & 99.8 \\
\hline
\end{tabular}
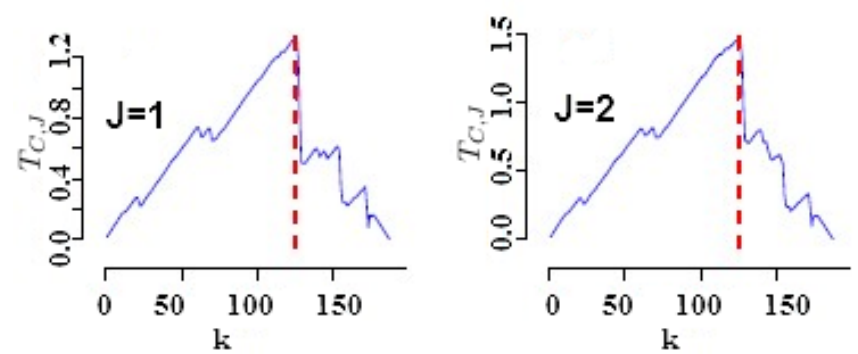

ciated to level $J=1$ depicts the same pick as the other series $T_{C, J}(k)$ for $J>1$ but its maximum value is less than the critical value $q_{\alpha}=1.358$ and fails to reject $H_{0}$. Estimation of the break point $\hat{k}$ based on the statistic $T_{N}$ suggests that a change occured at the point 126 which correspond to the second quarter of the year 2001 as indicated by the vertical dashed line in figure 2 . The estimator of the change point $k_{J}$ based on $T_{C, J}(k)$ is obtained as follows $\hat{k}_{J}=\operatorname{argmax} T_{C, J}(k)$, and the computed value for $J=2,3,4$ is $\hat{k}_{J}=125$ which correspond to the same quarter of 2001.

\section{Conclusions}

The wavelet based statistic $T_{C, J}$ gives us a scale by scale evaluation test of the homogeneity of the variance of $X_{t}$ using the classical CUSUM based test designed to detect a change in the mean of a time series which correspond to the cumulative wavelet coefficients $\widetilde{C}_{J, t}$ in our sitting. The over all performance in term of rejection rate of the statistic based on our simulation are as good as the tests in Inclan [8] like tests. This is comfirmed by the processing of real data given by the US personal savings series and support the practical utility of this statistic. The graph of the series $X_{t}$ in the top panel of figure 2 depicts a high variability after the year 2000 and this is detected by both statistics. Table 3 shows that the scale related statistics $T_{C, J}$ are doing quite well except for $J=1$ which just barely fail to reject the $H_{0}$. With regard to the identification of the change point both statistic estimate agree that the change occurs over the second quarter of the year 2001. Our wavelet based statistic is effective and this is mainly due to the attractive property of wavelets coefficients which demean and detrend time series. Its important to note here that because the wavelets demean the series, we can safely exclude any changes in the variance due to the mean. This study shows that our

Figure 3. Plot of the statistic $T_{C, J}(k) / \hat{\sigma}_{Z}$ for $J=1,2,3,4$ for the US personnal savings. The vertical dashed lines are the identified break locations 
Table 3. Computed test statistics $T_{C, J}$ and $T_{N}$ for the US Savings series

\begin{tabular}{|c|c|c|c|c|}
\hline$T_{C, 1}$ & $T_{C, 2}$ & $T_{C, 3}$ & $T_{C, 4}$ & $T_{N}$ \\
\hline 1.314 & 1.45 & 1.493 & 1.501 & 1.475 \\
\hline
\end{tabular}

nonparametric method provide a reliable statistic that can be applied to a broad class of time series data.

\section{Appendix}

\section{Proof of lemma 1}

$E\left(C_{J, t}\right)=\sum_{j=1}^{J} E\left(W_{j, t}^{2}\right)=\sum_{j=1}^{J} \sigma_{W_{j}}^{2}$.

Then $E\left(Z_{J, t}^{2}\right) \leq 2 E\left|C_{J, t}\right|^{2}+2\left(\sum_{j=1}^{J} \sigma_{W_{j}}^{2}\right)^{2}$.

On the other hand it follows from Holder's inequality with $p=\beta$ and $q=p /(p-1)$

$E\left|C_{J, t}\right|^{\beta}=E\left|\sum_{j=1}^{J} W_{j, t}^{2}\right|^{\beta} \leq J^{\beta / q} E\left(\sum_{j=1}^{J}\left|W_{j, t}^{2}\right|^{p}\right)^{\beta / p}=$ $J^{\beta-1} \sum_{j=1}^{J} E\left|W_{j, t}\right|^{2 \beta}$

In a similar manner we can easily show that $E\left|W_{J, t}\right|^{\beta} \leq$ $K_{j}(\beta) \sum_{l=0}^{L_{j}-1} E\left|X_{t-l}\right|^{\beta}$ and

$$
\begin{aligned}
\sup _{t} E\left|C_{J, t}\right|^{\beta} & \leq J^{\beta-1} \sum_{j=1}^{J} K_{j}(\beta) L_{j} \sup _{t} E\left|X_{t}\right|^{\beta} \\
& =J^{\beta-1} B_{J}(\beta) \sup _{t} E \mid X_{t}^{\left.\right|^{\beta}}
\end{aligned}
$$

where $K_{j}(\beta)$ is a constant and $\sum_{j=1}^{J} K_{j}(\beta) L_{j}=B_{J}(\beta)$.

Using Minkowski inequality $\left|Z_{J, t}\right|^{\beta} \leq 2^{\beta-1}\left(\left|C_{J, t}\right|^{\beta}+\left|\mu_{C_{J}}\right|^{\beta}\right)$ it follows that $\sup E\left|Z_{J, t}\right|^{\beta}<\infty$ and then (10)

Let $Y_{j, t}=W_{j, t}^{t}-E\left(W_{j, t}^{2}\right)$, then it follows from proof of theorem 1 in [17], page 195 that $\alpha_{Y_{j}}(k) \leq \alpha_{W_{j}}(k)$ for any $1 \leq j \leq J$. Therefore (11) follows and

$$
\alpha_{Z_{J}}(k) \leq J \max _{j \leq J} \alpha_{W_{j}}(k)
$$

On the other hand, it follows from proof of lemma 1 in [17], page 195

$$
\alpha_{W_{j}}(k) \leq \alpha_{X}\left(k-L_{j}-1\right) .
$$

and $\left.\sum_{k=1}^{\infty}\left(\alpha_{W_{j}}(k)\right)^{\gamma} \leq \sum_{k=1}^{L_{j}-1}\left(\alpha_{W_{j}}(k)\right)^{\gamma}+\sum_{n=1}^{\infty} \alpha_{X}(n)\right)^{\gamma}<\infty$

with $\gamma=1-2 / \beta$. Therefore

$$
\sum_{n=1}^{\infty}\left(\alpha_{Z_{J}}(k)\right)^{\gamma} \leq J^{\gamma} \sum_{n=1}^{\infty}\left(\max _{j \leq J} \alpha_{W_{j}}(k)\right)^{\gamma}<\infty
$$

The result (12) follows then from lemma 2.

Lemma 2

Let $\left\{X_{t}\right\}$ be a sequence of random variables with $E\left(X_{t}\right)=0$

and satisfying for $\beta>2$ :

(C1) $\sup _{t}\left|X_{t}\right|^{\beta}<\infty$

(C2) $\left\{X_{t}\right\}$ is $\alpha$-mixing such that $\sum_{n=1}^{\infty}\left(\alpha_{X}(n)\right)^{1-2 / \beta}<\infty$

Then (i) $E\left(\frac{1}{n}\left(\sum_{t=1}^{n} X_{t}\right)^{2}\right) \rightarrow \sigma_{0}^{2}<\infty$ and

(ii) limsup $\left\|X_{t}\right\|_{\beta}<\infty$

Proof. From lemma 2 of Herrndorf [5], it follows that

$$
\begin{aligned}
E\left(\frac{1}{n}\left(\sum_{t=1}^{n} X_{t}\right)^{2}\right) & =\frac{1}{n} \sum_{t=1}^{n} E\left(X_{t}^{2}\right)+\frac{2}{n} \sum_{1 \leq t<k \leq n} E\left(X_{t} X_{k}\right) \\
& \leq \frac{1}{n} \sum_{t=1}^{n} E\left(X_{t}^{2}\right)+\sum^{\frac{2}{n}} \sum_{1 \leq t<k \leq n}^{\gamma}\left(\alpha_{X}(|t-k|)\right)^{\gamma}\left\|X_{t}\right\|_{\beta}\left\|X_{k}\right\|_{\beta}
\end{aligned}
$$

with $\gamma=1-2 / \beta$.

Now $\left\|X_{t}\right\|_{\beta}\left\|X_{k}\right\|_{\beta} \leq\left(\sup _{t}\left\|X_{t}\right\|_{\beta}\right)^{2}=C_{X}(\beta)$. Then

$$
\begin{aligned}
\left|\sum_{1 \leq t<k \leq n} E\left(X_{t} X_{k}\right)\right| & \leq C_{X}(\beta) \sum_{1 \leq t<k \leq n}\left(\alpha_{X}(|t-k|)\right)^{\gamma} \\
& =C_{X}(\beta) \sum_{k=1}^{n=1}(n-k)\left(\alpha_{X}(k)\right)^{\gamma}
\end{aligned}
$$

Therefore

$$
\begin{aligned}
E\left(\frac{1}{n}\left(\sum_{t=1}^{n} X_{t}\right)^{2}\right) & \leq \frac{1}{n} \sum_{t=1}^{n} E\left(X_{t}^{2}\right) \\
& +2 C_{X}(\beta) \frac{1}{n} \sum_{k=1}^{n-1}(n-k)\left(\alpha_{X}(k)\right)^{\gamma}
\end{aligned}
$$

which converges to $\sigma_{0}^{2}$ as $n \rightarrow \infty$.

Note that if $\left\{X_{t}\right\}$ is a second order stationary process with mean zero and variance $\sigma_{X}^{2}$, then

$$
\begin{aligned}
E\left(\frac{1}{n}\left(\sum_{t=1}^{n} X_{t}\right)^{2}\right) & =\frac{1}{n} \sum_{t=1}^{n} \sigma_{X}^{2}+\frac{2}{n} \sum_{k=1}^{n-1}(n-k) s_{X, k} \\
& =\sigma_{X}^{2}+\frac{2}{n} \sum_{k=1}^{n-1}(n-k) s_{X, k} \\
& =\sigma_{X}^{2}+\sum_{k=1}^{n-1}\left(1-\frac{k}{n}\right) s_{X, k} \underset{n \rightarrow \infty}{\rightarrow} \sigma_{0}^{2}
\end{aligned}
$$

On the other hand $\left.E\left(\frac{1}{n}\left(\sum_{t=1}^{n} X_{t}\right)^{2}\right)=n E\left(\left(\bar{X}^{(n)}\right)^{2}\right)\right)=$ $n \operatorname{Var}\left(\bar{X}^{(n)}\right) \rightarrow S_{X}(0)$ where $S_{X}(0)$ is the sdf of $X$ at 0 frequency. Hence $\sigma_{0}^{2}=S_{X}(0)$

(ii) Note that $\limsup _{n}\left\|X_{t}\right\|_{\beta}=\limsup _{n}\left\|X_{t \leq n}\right\|_{\beta}<\infty, \beta>2$.

The result follows readly from condition $(\mathrm{C} 1)$.

\section{REFERENCES}

[1] Coifman, R. and Donoho, D. L. Translation-invariant Denoising In: Wavelet and Statistics: Lecture Notes in Statistics. edited by Antoniadis, A. Oppenheim, G., New York: Springer-Verla, vol. 103, 125-50, 1995.

[2] Daubechies, I. Ten Lectures on wavelets, Philadelphia: SIAM, 1992.

[3] Donald B. Percival, and Andrew T. Walden. Wavelet Methodes for Time Series Analysis, Cambridge Series in Statistical and Probabilistic Mathematics, 2000.

[4] Douglas M. Hawkins, K. D. Zamba. A Change Point Model for a Shift in Variance, Journal of Quality Technology, Vol. 37, No.1, 21-31, 2005. 
[5] Herrndorf, N. f, C. A functional Central Limit Theorem for Weakly Dependentt Sequences of Random Variables, Annals of Probability, 12, 141-153, 1984.

[6] Horv'ath L., P. Kokoszka, and G. Teyssi'ere, Empirical Process of the Squared Residuals of an ARCH Sequence, Annals of Statistics, 29, 445-469, 2001.

[7] Hsu, D. A. Tests for Variance Shift at an Unknown Time Point, Journal of the Royal Statistical Society. Series C, Applied Statistics, Vol. 26, No3, 279-284, 1977.

[8] Inclan, C. and G. C. Tiao. Use of Cumulative Sums of Squares for Retrospective Detection of Changes of Variance, Journal of the American Statistical Association, 89 (427), pp. 913-923, 1994.

[9] Kokoszka, P. and R. Leipus. Change-point Estimation in ARCH Models, Bernoulli, 6, 513-539, 2000.

[10] R. Killick, I. A. Eckley, A wavelet-based approach for detecting changes in second order structure within nonstationary time series, Electronic Journal of Statistics, Vol. 7, 1167-1183, 2013.

[11] Michiel de Pooter and Dick van Dijk, Testing for Changes in Volatility in Heteroskedastic Time Series - A Further Examination, Econometric Institute Research Papers, No EI 2004-38.
[12] Nason, G.P. and Silverman, B.W. The stationary Wavelet Transform and Some Statistical Applications, Wavelet and Statistics: Lectures Notes in Statistics. edited by Antoniadis, A. Oppenheim, G., New York: Springer-Verla, Vol.103, 281-99, 1995.

[13] Ploberger, W. and W. Kramer. The CUSUM Test with OLS Residuals, Econometrica, 60(2), 271-85, 1992.

[14] Rosenblat, M. Stationary Sequences and Random Fields. Boston, Birkhauser, 1985.

[15] Sangyeol Lee, Okyoung Na. and Seongryongna. On the Cusum of squares test for variance changes in nonstationary and nonparametric time series models, Ann. Inst. Statist. Math., Vol. 55, No. 3, 467-485, 2003.

[16] Serroukh A. and A. T Walden. Wavelet Scale Analysis of Bivariate Time Series I: Motivation and Estimation, Nonparametric Statistics, Vol. 13, pp. 1-36, 2000.

[17] Serroukh A. \& A. T. Walden, and D.B. Percival. Statistical Properties and uses of Wavelets Variance Estimator for the Scale Analysis of Time Series, Journal of the American Statistical Association, Theory and Methods, Vol. 95 N0 449, 2000. 